Published in final edited form as:

Nat Struct Mol Biol. 2018 May ; 25(5): 384-393. doi:10.1038/s41594-018-0057-1.

\title{
Mapping Interactions with the Chaperone Network Reveals Factors that Protect Against Tau Aggregation
}

\author{
Sue-Ann Mok ${ }^{1}$, Carlo Condello ${ }^{1}$, Rebecca Freilich ${ }^{2}$, Anne Gillies $^{2}$, Taylor Arhar ${ }^{2}$, Javier \\ Oroz $^{6}$, Harindranath Kadavath ${ }^{6}$, Olivier Julien², Victoria A. Assimon ${ }^{2}$, Jennifer N. Rauch ${ }^{2}$, \\ Bryan M. Dunyak ${ }^{2}$, Jungsoon Lee $^{3}$, Francis T.F. Tsai ${ }^{3}$, Mark R. Wilson ${ }^{4}$, Markus \\ Zweckstetter ${ }^{5,6,7}$, Chad A. Dickey ${ }^{8}$, and Jason E. Gestwicki ${ }^{1,2,{ }^{*}}$ \\ ${ }^{1}$ Department of Neurology, University of California at San Francisco, San Francisco, CA, 94158, \\ USA \\ ${ }^{2}$ Department of Pharmaceutical Chemistry, University of California at San Francisco, San \\ Francisco, CA, 94158, USA \\ ${ }^{3}$ Department of Biochemistry and Molecular Biology, Baylor College of Medicine, Houston, TX, \\ 77030, USA \\ ${ }^{4}$ llawarra Health and Medical Research Institute, School of Biological Sciences, University of \\ Wollongong, Wollongong, New South Wales, Australia \\ ${ }^{5}$ Max-Planck-Institut für Biophysikalische Chemie, 37077 Goettingen, Germany \\ ${ }^{6}$ Deutsches Zentrum für Neurodegenerative Erkrankungen (DZNE), von-Siebold-Str. 3a, 37075 \\ Göttingen, Germany \\ ${ }^{7}$ Department of Neurology, University Medical Center Göttingen, University of Göttingen, \\ Waldweg 33, 37073 Göttingen, Germany \\ ${ }^{8}$ Department of Molecular Medicine and Byrd Alzheimer's Research Institute, University of South \\ Florida, Tampa, Florida 33613, USA
}

\section{Abstract \\ A network of molecular chaperones is known to bind proteins ("clients") and balance their folding, function and turnover. However, it is often not clear which chaperones are critical for selective recognition of individual clients. It is also not clear why these key chaperones might fail in protein aggregation diseases. In this study, we utilized human microtubule-associated protein tau (MAPT}

Users may view, print, copy, and download text and data-mine the content in such documents, for the purposes of academic research, subject always to the full Conditions of use: http://www.nature.com/authors/editorial_policies/license.html\#terms

*Correspondence can be addressed to: jason.gestwicki@ucsf.edu.

Author contributions:

S.A.M.- Conception and design, Acquisition of data, Analysis and interpretation of data, Drafting and revising the article

R.E.F, C.C., J.O., H.K.- Acquisition of data, Analysis and interpretation of data

A.G., T.A. - Acquisition of data

O.J. - Analysis and interpretation of data

N.J., V.A., B.D., J.R. J.L.- Contributed reagents

F.T.F.T., M.W., M.Z. - Contributed reagents, Drafting or revising the article

C.A.D. -Conception and design

J.E.G. - Conception and design, Drafting and revising the article 
or tau) as a model client to survey interactions between $\sim 30$ purified chaperones and $\sim 20$ diseaseassociated tau variants ( $\sim 600$ combinations). From this large-scale analysis, we identified human DnaJA2 as an unexpected, but potent, inhibitor of tau aggregation. DnaJA2 levels were correlated with tau pathology in human brains, supporting the idea that it is an important regulator of tau homeostasis. Of significance, we found that some disease-associated tau variants were relatively immune to interactions with chaperones, suggesting a model in which avoiding physical recognition by chaperone networks may contribute to disease.

Impact Statement-Large-scale screening of chaperone interactions with tau and its variants identified DnaJA2 as a key protective factor in tauopathy.

\section{Major subject areas keywords and research organism(s)}

Biochemistry; Neuroscience; Proteostasis; protein folding; protein aggregation; protein-protein interactions; tauopathy; heat shock proteins; protein quality control; purified proteins

\section{Introduction}

The chaperone network is composed of "core" chaperones, such as heat shock protein 70 (Hsp70) and heat shock protein 90 (Hsp90), that utilize nucleotide hydrolysis to power reversible interactions with client proteins ${ }^{1}$. These core chaperones are further assisted by co-chaperones that help decide which clients should be bound and whether they should be folded, trafficked or degraded. Together, the entire human chaperone and co-chaperone network is composed of $\sim 332$ gene products (i.e. the "chaperome") ${ }^{2}$. However, the individual components of the human chaperone network do not act in isolation; rather, they are known to engage in widespread protein-protein interactions between themselves and with clients ${ }^{3,4}$. Moreover, this chaperone network is part of a broader cellular system, including translation control and the stress responses, that work together to maintain protein homeostasis (i.e. proteostasis) ${ }^{1,5-7}$.

Still, this robust, conserved system does fail. For example, a shared hallmark of neurodegenerative diseases is the aberrant misfolding and aggregation of proteins ${ }^{8}$. Often, the pathology of these diseases is dominated by the aggregation of a single protein in the form of large, fibrillary deposits. Why does the normal proteostasis system fail in these cases? Several studies have found that the levels of many chaperones and co-chaperones are reduced during aging $2,9,10$, which likely diminishes the overall ability of a cell to maintain proteostasis. In further support of this idea, activation of longevity pathways in worms enhances chaperone expression and limits aggregation of amyloid beta and polyglutamine (polyQ) proteins ${ }^{9,10}$.

This general paradigm is compelling, but it does not directly explain why specific proteins can sometimes circumvent the chaperone network. For example, a missense mutation, $\mathrm{P} 301 \mathrm{~L}$, in the microtubule-associated protein tau (referred to as MAPT or tau) gives rise to the progressive, lethal neurodegenerative disease: frontotemporal dementia with parkinsonism linked to chromosome 17 (FTDP-17). Why does the chaperone network fail to effectively "handle" P301L tau? We hypothesized that individual mutant proteins may sometimes evade molecular recognition by specific components of the chaperone network. 
Consistent with this "evasion" model, genetic screens have shown that over-expression of certain "core" chaperones, such as Hsp70s, restores protection from aggregation of polyQ proteins ${ }^{11}$ and P301L tau ${ }^{12,13}$. However, Hsp70s are rather promiscuous factors. The cochaperones that might underlie selective, molecular recognition of tau and its variants have been less clear. This gap is understandable because partially redundant functions of cochaperones often make large-scale genetic screens for discovering them more challenging 14 and their direct interactions with clients are often exceptionally weak, which makes proteomics difficult.

In this study, we set out to identify chaperones and co-chaperones (from now on referred to collectively as chaperones) that directly bind to tau. We chose tau for this effort because accumulation of aggregated tau is associated with more than a dozen neurodegenerative disorders, including FTDP-17, mild cognitive impairment (MCI) and Alzheimer's disease (AD) ${ }^{15}$. Moreover, tau is an intrinsically disordered protein (IDP), which removes the potential complication of controlling for folding-unfolding transitions. Finally, tau is an established chaperone client and individual chaperone interactions (i.e. positive controls) have been reported ${ }^{16-18}$. Based on these criteria, we established a series of complementary in vitro and cellular screening paradigms to identify the subset of chaperones that might bind tau and prevent its aggregation. In this effort, we studied the interactions of $\sim 30$ major chaperones with $\sim 20$ disease-associated variants of tau to understand why or how mutations and PTMs might influence chaperone activities. From these screens, we made the unexpected discovery that the chaperone, DnaJA2, was a strong inhibitor of tau aggregation and that it was active against a relatively broad number of tau variants. Furthermore, in patients with mild cognitive impairment (MCI) we found a striking upregulation of DNAJA2 that was specific to neurons positive for markers for tau and amyloid pathology. This work showcases how a large-scale, biochemical approach to map direct interactions of clients with chaperones might complement other methods (e.g. genomics, proteomics) to reveal important recognition events.

\section{Results}

\section{Select chaperones modulate tau aggregation kinetics and these interactions are sensitive to disease-associated modifications}

To date, only a very limited number of chaperones (Hsc70, Hsp27, FKBP51) have been examined, in isolation, for their ability to modulate the aggregation of tau ${ }^{12,19,20}$. We set out to greatly expand this dataset by comparative analysis of $>30$ chaperones for their effects on tau aggregation kinetics. Tau is subject to splicing to produce multiple isoforms ${ }^{16}$. These splice isoforms include a variable number of $\mathrm{N}$-terminal repeats $(0 \mathrm{~N}-2 \mathrm{~N})$, a polyproline region and either three or four microtubule binding repeats (MBRs; R1-R4) (Fig. 1a). An important feature of the MBRs is the presence of two aggregation motifs: termed PHF6* (275-VQIINK-280) and PHF6 (306-VQIVYK-311), which are required for aggregation $21-23$. Tau aggregation is typically measured using Thioflavin (ThT), a dye that binds $\beta$ sheets. For our studies, we miniaturized the ThT assay for use in 384-well plate format to: (a) facilitate a greater number of replicates and (b) test multiple concentrations of each chaperone. To validate this platform, we first titrated a major constituitively expressed 
member of the Hsp70 family, Hsc70 (heat shock cognate 70, HSPA8), into solutions of purified $0 \mathrm{~N} 4 \mathrm{R}$ tau ${ }^{\mathrm{WT}}$ and measured the kinetics of aggregation after addition of the commonly-used accelerant, heparin. We selected 0N4R tau for these studies because it is a major splice isoform in adult brain and has relatively rapid aggregation kinetics. Consistent with previous reports ${ }^{20}$, we found that purified Hsc70 increased the lag time and suppressed the elongation rate of tau aggregation reactions (Fig. 1a; Supp. Fig. 1a). In contrast, a negative control construct of Hsc70 encompassing only the nucleotide binding domain $\left(\mathrm{Hsc}_{\mathrm{NBD}}\right)$ had relatively little effect on aggregation kinetic parameters.

Next, we purified 18 variants of $0 \mathrm{~N} 4 \mathrm{R}$ tau to test in this format. These variants included disease-associated mutations (P301L, A152T, $\Delta \mathrm{K} 280$ ) and mimics of disease-associated phosphorylation (T153E, T231E, S356E, S396E, S404E), acetylation (K174Q, K280Q, K281Q), and caspase-cleaved fragments (D25, D421) (Fig. 1b and Supp. Table 1). The collection also included variants in which the individual aggregation motifs were disrupted (the natural 0N3R tau isoform and a 0N4R $\Delta 308 / 309$ construct), variants with mutations in known proline isomerization motifs (P232A, T,P231E,A) as well as the widely-studied, synthetic tau construct, termed K18, that includes the four MBRs. It is known that some of these modifications either accelerate or slow tau aggregation kinetics in vitro ${ }^{21,23}$, so we first characterized the kinetics of each variant using the miniaturized ThT assay. Most of the ON4R tau variants had lag times (Fig. 1c) and elongation rates (Supp. Fig. 1b) similar to 0N4R tau ${ }^{\mathrm{WT}}$, with the exception of the disease-associated mutants: $\Delta \mathrm{K} 280$ and P301L, which aggregated significantly faster. As expected, the tau variants WT 0N3R tau and $\Delta 308 / 309$, which lack the PHF6 motif, had 5- to 10-fold longer lag phases and 2-fold slower elongation rates. These baseline studies allowed us to normalize subsequent results to the intrinsic aggregation propensity of each variant.

Each tau variant was then tested against a purified human chaperone collection. The collection contained major members of the Hsp70 and small heat shock protein (sHsp) chaperone systems. The throughput of the ThT platform also allowed us to test additional chaperone previously linked to tauopathy, including clusterin, aB-crystallin, FKBP12 and Pin1 ${ }^{24-27}$ as well as chaperones known to have roles in other protein-misfolding diseases, such as Hsp60 and HIP ${ }^{28,29}$. In addition, we assayed a select number of anti-aggregation chaperones from other organisms, including yeast $\mathrm{Hsp} 104^{30}$ and several newly-discovered chaperones from Escherichia coli and Saccharomyces cerevisae ${ }^{31,32}$. For each experiment, the purified chaperone was incubated with tau $(10 \mu \mathrm{M})$ prior to addition of heparin. In addition, each chaperone was tested in triplicate at three concentrations within the range of 2.5 to $20 \mu \mathrm{M}$ to probe whether effects on aggregation were reproducible and dosedependent. Replicates of the screen demonstrated good correlation and day-to-day reproducibility (Supp. Fig. 1c-d), giving us confidence in the results. In addition, the negative control (Hsc70 ${ }_{\mathrm{NBD}}$ ) was inactive (Fig. 1d), except for some modest, non-specific activity against the slowly aggregating $0 \mathrm{~N} 3 \mathrm{R}$ and $\Delta 308 / 309$ variants.

Incubation of $\sim 30$ chaperones with $\sim 20$ tau variants revealed a fascinating pattern of effects on tau lag time (Fig. 1d) and elongation rate (Supp. Fig. 2a). For clarity, the effects at 1:1 stoichiometry (tau:chaperone) are shown, but similar results, albeit of lower magnitude, were seen at reduced concentrations (Supp. Fig. 2b). The first striking observation is that a 
subset of chaperones, including Hsc70, DnaJA2, Hsp60, clusterin, and Hsp104, were the most potent suppressors of tau aggregation. Interestingly, these chaperones share little similarity in terms of their chaperone family or their structure. Despite these differences, some of them are known to have anti-aggregation activity against other clients ${ }^{31,33,34}$ or function as protein disaggregases ${ }^{35}$. The exceptions were Hsp60 and DnaJA2, which had not previously been linked to aggregation or tau biology. DNAJA2 is closely related to another J-protein member, DNAJA1. DNAJA1 could not be tested using the screen conditions due to issues with solubility however, a modified tau aggregation assay showed that DNAJA1 was also able to inhibit $0 \mathrm{~N} 4 \mathrm{R}$ tau ${ }^{\mathrm{WT}}$ aggregation (Supp. Fig. 2c). A construct of the conserved $\mathbf{J}$ domain, taken from E.coli DNAJ, had minimal effects on tau aggregation for most of the tau variants in the screen (Fig. 1d), consistent with the idea that substrate binding is often independent of the J-domain in this family of co-chaperones.

Another striking observation from this analysis was that certain tau variants, such as P301L, A152T, $\Delta K 280, D 25$ and D421, were relatively resistant to even the most potent chaperones (Fig. 1d and Supp. Fig. 2d). To extend this observation, we tested several hit chaperones against additional disease-associated mutations (Fig. 1f). Consistent with the original observations, the aggregation of $\mathrm{N} 279 \mathrm{~K}$ and P301S mutants was also relatively resistant to chaperone protection when compared to WT ON4R tau. This result suggests that some tau variants might partially, but broadly, circumvent the anti-aggregation activities of chaperones. It is also worth noting that the tau variants lacking one aggregation motif (0N3R and $\Delta 308 / 309$ ) were protected by a wider range of chaperones (Fig. 1d). It is known that an imbalance in the ratio of $4 \mathrm{R}$ tau vs. $3 \mathrm{R}$ tau is linked to some tauopathies ${ }^{36}$. Our results suggest that the switch towards $4 \mathrm{R}$ isoforms may make tau aggregation more resistant to chaperones.

One of the unexpected results of this analysis was the identification of chaperones that only suppressed the aggregation of a few tau variants. For example, the small HSPs (Hsp22, Hsp27, aB-crystallin) delayed aggregation of several tau phosphomimetics (e.g. S356E) but not the others. Another unexpected result was the difference in activity between the two closely related Hsp70 family members, Hsc70(HSPA8) and Hsp72(HSPA1A). Overall, Hsc70 was a much better anti-aggregation chaperone, even though it is $94 \%$ identical to Hsp72 and both Hsc70 and Hsp72 bind to tau with similar affinity ${ }^{37}$. We generated analogous constructs of the isolated Hsc70 and Hsp72 substrate binding region consisting of the $\beta$-sheet substrate-binding cleft and the first two a-helices of the lid (aa 394-540). At the highest concentrations tested $(20 \mu \mathrm{M}), \mathrm{Hsc} 70_{\mathrm{SBD}}$ and $\mathrm{Hsp} 72_{\mathrm{SBD}}$ delayed the aggregation of $0 \mathrm{~N} 4 \mathrm{R}$ tau $^{\mathrm{WT}}$ to the same degree as their corresponding full-length proteins. These results suggest that the reduce anti-aggregation activity of Hsp72 can likely be attributed to the relatively few residues that differ between Hsc70 and Hsp72 in the SBD. Future studies exploring the isolated SBDs may help to shed light on the differential actions of $\mathrm{Hsc70}$ and Hsp72 on tau homeostasis observed previously 37,38 .

Finally, we were surprised to identify chaperones that promoted aggregation. For example, FKBP12 had no effect on the aggregation of $0 \mathrm{~N} 4 \mathrm{R}$ tau ${ }^{\mathrm{WT}}$ but it promoted aggregation of the acetylation mimetic, K280Q. These results support a model that, in some contexts, individual chaperones might facilitate tau pathology 19 . 


\section{Some chaperones suppress lag time, while others inhibit elongation rate}

While we initially focused on the effects of chaperones on lag time, our screen captured other valuable data regarding elongation rate effects. Studies on amyloid beta (A $\beta$ ) have been informative in elucidating the microscopic processes occurring during different phases of an amyloid aggregation reaction ${ }^{39}$. From that work, stages, such as primary nucleation, secondary nucleation and elongation of fibril ends, all seem to contribute to the reaction. Although, a parallel study on tau aggregation has not yet been reported, we hypothesized that differential effects of individual chaperones in lag time and elongation rate might be suggestive of the stage at which the chaperone was acting ${ }^{41}$. When profiling chaperones for their effects on aggregation kinetics across $0 \mathrm{~N} 4 \mathrm{R}$ tau variants we observed that the chaperones Hsc70, Hsp60 and clusterin, greatly slowed both lag time and elongation rate (Fig. 2a). However, DnaJA2 and Hsp104 were less active against tau elongation (relative to their effects on lag time), while Hsp27 and aB-crystallin were significantly more potent on the elongation step. These unique signatures suggested that individual chaperones might supress only a subset of microscopic processes in the aggregation reaction. Thus, we reasoned that combining chaperones with distinct activity profiles could lead to synergistic effects. To test this idea, we assayed select, pairwise combinations of chaperones (1:1 ratio) for their effects on tau lag time kinetics. In this analysis, we asked whether the effects on lag time when two chaperones were present $(5 \mu \mathrm{M}$ each) would be greater than the sum of the effect observed for each chaperone when tested alone $(5 \mu \mathrm{M})$. In general, we found that combining two chaperones led to antagonistic, not synergistic, effects on anti-aggregation activity (Fig. 2b). For example, combining Hsp72 with DnaJA2 or Hsp60, led to lower than predicted increases in lag time. This result might occur when chaperones compete for the same tau binding sites (see next section). Interestingly, a small subset of combinations showed synergistic activity, including DnaJA2 with Hsp22 or aB-crystallin, suggesting that these chaperones act by cooperative mechanisms.

Next, we wanted to probe whether any of the chaperones could still suppress tau aggregation once the reaction had already been initiated. Accordingly, we added individual chaperones at 0,25 , or 55 minutes post-induction. We found that delaying the addition of chaperone until 25 min into the aggregation reaction (during the lag time) still extended lag times (Fig. 2c). However, we noticed that the magnitude of the effect was smaller when compared to addition at time zero or under screen conditions (i.e. addition of chaperone at $-30 \mathrm{~min}$ ). Delaying the addition of the chaperone until 55 minutes into the aggregation reaction (at the beginning of the elongation phase), largely abolished anti-aggregation activity of chaperones (Fig. 2c). Only DnaJA2 was able to significantly decrease the rate of aggregation under these conditions. Surprisingly, several chaperones (i.e. aB crystallin, Hsp27, and clusterin) enhanced aggregation rates when added post-lag phase, despite having the opposite effect when added under the screening conditions. Thus, from these studies, it seems likely that chaperones can affect multiple processes in the tau aggregation pathway ${ }^{41}$.

One trivial reason for DnaJA2's anti aggregation properties might be that it binds to the accelerant heparin. To test this possibility, we induced tau aggregation with an un-related accelerant, arachidonic acid, and found that DnaJA2 retains its ability to suppress aggregation (Supp. Fig. 3a). Moreover, although DnaJA2 had some, weak affinity ( $2 \mu \mathrm{M})$ 
for heparin in vitro (Supp. Fig. 3b), increasing heparin levels did not abolish DnaJA2's antiaggregation activity (Supp. Fig. 3c).

Finally, we tested whether chaperones could disassemble mature fibrils (incubated $>20 \mathrm{hrs}$ ), as measured by a decrease in ThT signal. The Bukau group pioneered studies of chaperone complexes that have disaggregase activity on a-synuclein amyloids ${ }^{42}$. However, we found that none of the chaperones, by themselves, were able to reverse aggregation of 0N4R tau WT fibrils (data not shown, S.A.M, J.E.G.). This result is not surprising, as the Bukau group showed that only combinations of chaperones, plus ATP, creates disaggregase activity. We have not yet identified the combination of chaperones and ATP that might act as a putative disaggregase for tau fibrils.

\section{Chaperones bind to hotspots within the tau polypeptide}

Our screen results prompted us to ask where chaperones might bind on tau. Specifically, we wondered which chaperones might bind directly to the PHF6 and PHF6* regions in the MBRs that are known to be required for tau aggregation. Previous studies have mapped where one or two chaperones bind to individual clients in vitro ${ }^{43-46}$, but little is known about client recognition in a broader context. Peptide arrays have previously been used to map the binding sites of Hsp70s and Hsp90a ${ }^{17}$ on tau and these interactions sites have been confirmed and extended by NMR spectroscopy ${ }^{37,45}$. Inspired by these approaches, we mapped the binding sites on tau for our broader collection of chaperones. Briefly, a tau peptide array was generated with 15-mer peptides in 4 amino acid steps that covered the longest adult isoform of tau, 2N4R. We then prepared a set of eighteen His-tagged purified chaperones that included several chaperones from the aggregation screen that were compatible with the solubility and tagging requirements of the platform. The binding of the His-tagged chaperones $(10 \mu \mathrm{M})$ was measured with fluorescently-labeled anti-His antibodies. After removing false positives that bound anti-His antibody and/or Hsp70 $0_{\mathrm{NBD}}$, we annotated true binding sites as regions composed of at least two, contiguous 15-mer peptides (Fig. 3a, also see Methods). We found that previously assayed chaperones, Hsp72 and Hsp90a, bound sites overlapping both aggregation motifs, PHF6* (aa 275-280) and PHF6 (aa 3-311) and near the polyproline-R1 boundary (aa 220-242) (Supp. Fig. 4a and b), as expected ${ }^{17}$. Strikingly, we found that a number of other chaperones, including DnaJA1, DnaJA2, Hsp27 and FKBP51 also bound peptides in or around PHF6* and PHF6 (Fig. 3a). We also noted that there were additional high frequency chaperone-binding sites in the polyproline region (aa 220-242) and the N-terminus (aa 5-23). These regions were also interesting because they correspond to multiple $\mathrm{AD}$-associated phosphorylation sites ${ }^{47}$, a known Pro232 isomerization site ${ }^{48}$, and an $\mathrm{N}$-terminal region previously linked to tauopathy 49. The peptides that bound to multiple chaperone families shared no overall similarity in characteristics such as charge, pI, aliphatic index, or hydrophobicity (Supp. Table 2), consistent with the relatively broad binding properties expected from these different structural classes.

Next, we carried out NMR studies on Hsp72, Hsp90, DnaJA2, Hsp27 and DnaJB4 by titrating them into solutions of ${ }^{15} \mathrm{~N}$-labeled WT $2 \mathrm{~N} 4 \mathrm{R}$ tau (Fig. 3a). This was an important experiment because peptide arrays cannot mirror the broader context of surrounding regions 
in polypeptides. Despite this complication, we found that the location of the interactions, as measured by NMR, generally supported the peptide array results. Specifically, Hsp72, Hsp90 and DnaJA2 seemed to bind regions that spanned the polyproline and MBRs (R1R4), aligning with previous NMR studies when available $\mathrm{e}^{37,45}$. However, compared to the peptide array, the NMR-based study also picked up broader footprints for Hsp72, Hsp90 and DnaJA2, which often encompassed the R3 region containing the PHF6 motif. Interestingly, no binding was observed for Hsp27 suggesting that it may modulate tau aggregation through recognition of non-monomeric forms of the protein.

To further explore the interaction of anti-aggregation chaperones with the PHF6 and PHF6* regions of tau we carried out further NMR studies. Specifically, we titrated Hsc70, DnaJA2, clusterin, and Hsp60 into solutions of ${ }^{15} \mathrm{~N}$-labeled WT K18 tau (Fig. 3b). Owing to the higher resolution of this construct, we observe more discrete binding footprints. Specifically, we found that Hsc70, DnaJA2, clusterin, and Hsp60 bound to the region overlapping the PHF6 motif, along with weaker additional binding to the PHF6* region. Thus, a major mechanism of anti-aggregation activity seems to be direct binding to tau in the aggregationprone sequences.

\section{A subset of chaperones suppresses formation of tau "seeds"}

Recent studies have demonstrated that tau aggregates can have prion-like activity, including the ability to induce aggregation of soluble tau when introduced to cells ${ }^{50,51}$. Indeed, propagation of aggregates may be a potential mechanism for disease progression in a variety of neurodegenerative diseases ${ }^{52}$. Because we had found that several chaperones slowed tau aggregation, we next wondered whether this activity would suppress prion-like character. In support of this idea, centrifugation $(100,000 \mathrm{~g})$ of the tau samples co-incubated with chaperones showed that some, such as Hsc70, Hsp72 and DnaJA2, increased tau solubility (Supp. Fig. 5a and b). However, even small, soluble tau species have been determined to be capable of nucleating aggregation, so we did not consider the ability to block overall aggregation a sign of anti-prion-like activity ${ }^{53}$. Accordingly, we used a 'clone 1' cell line developed in the Diamond laboratory, in which K18 tau is stably expressed as a fusion to yellow fluorescent protein $(\mathrm{K} 18-\mathrm{YFP})^{54}$. In this model, the K18-YFP is distributed evenly in the cytoplasm but forms fluorescent puncta upon transfection with aggregated tau from patient brain or recombinant sources (Fig. 4a). Importantly, tau seeds can be titrated into this system and the number of cells with puncta quantified (Fig. 4b). As a control, we first made sure that none of the individual chaperones reduced the number of puncta when added to pre-formed fibrils (Supp. Fig. 5c). This result suggested that the chaperones themselves did not reduce aggregation when added exogenously. Then, to see if chaperones could suppress seeding activity, we transfected clone 1 cells with unfractionated 0 N4R tau ${ }^{\text {WT }}$ fibrils formed in the presence of individual chaperones (1:1 ratio), mirroring the initial ThT assay conditions. Strikingly, we found that Hsc70 and DnaJA2 were the only chaperones effective at reducing tau prions (Fig. 4c). DnaJA2 was particularly effective in this platform, and it even suppressed tau prions formed from P301L and A152T mutants. Thus, these results suggest that DnaJA2 likely acts on tau during the aggregation process to lower both its aggregation and its seeding potential. 


\section{DnaJA2 is upregulated in affected neurons of $\mathrm{MCl}$ and $\mathrm{AD}$ patients}

Our identification of DnaJA2 as a potent suppressor of tau aggregation in our in vitro studies led us to ask if DnaJA2 was linked to tau aggregate pathology in vivo. To test this idea, brain slices from patients with MCI or AD were labeled with antibodies for phospho-tau (AT8, green) and DnaJA2 (red). In addition, we used propidium iodide (PI) to indicate nuclei (blue) and FSB to stain amyloid (white). First, we confirmed that MCI samples had sparse tau pathology as marked by AT8 positivity (Supp. Fig 6a), while AD patients had greater pathology (Supp. Fig. 6b). In MCI samples, neurons positive for AT8 and/or FSB staining (Fig. 5a and b) had strikingly increased levels of DnaJA2 compared to surrounding neurons in the same sample or non-demented controls (Fig. 5c). Interestingly, the DnaJA2 staining does not co-localize with the AT8 or FSB signals in the soma or neurites (Fig. 5a and b) of affected MCI neurons; rather it was interspersed or surrounding the marked regions of pathology (Supp. Fig. 6c). Quantification of DnaJA2 levels showed increased staining for DnaJA2 in $80 \%$ of AT8 positive neurons in MCI samples (Fig. 5d) and $31 \%$ of AT8 positive neurons in $\mathrm{AD}$ (Fig. 5e). These results suggested that DnaJA2 might be a protective factor, that diminishes in $\mathrm{AD}$ neurons. We also noted that, in $\mathrm{AD}$ samples, there was prominent DnaJA2 staining at sites just adjacent to neurons, which are potentially surrounding glial cells (Supp. Fig. 6d).

Next, we compared the DnaJA2 results to staining with a control J-protein, DnaJB4. In contrast to our findings with DnaJA2, DnaJB4 levels remained unchanged between AT8 negative and positive neurons (Fig. 6f). Only 1 out of 18 AT8 positive neurons showed upregulation of DnaJB4 in MCI samples and no upregulation of DnaJB4 was observed in AD samples. Dot blot against purified recombinant proteins indicated that the DnaJA2 antibody was specific among a panel of other J-proteins and did not detect the closelyrelated family member, DnaJA1 (Supp. Fig. 6e). We were unable to validate a DnaJA1specific antibody for our study.

Finally, we examined the immunostaining patterns for several other anti-tau aggregation chaperones including Hsc70, Hsp72, and Hsp27. Hsc70 and Hsp72 staining patterns were not altered in AT8 positive neurons from MCI samples (Supp. Fig 6f and h) while Hsp27 was primarily observed in non-neuronal cells in non-demented controls (Supp. Fig. 6g), MCI (data not shown, C.C.) and AD samples (Supp. Fig 6g). In AD samples, Hsp27 staining was additionally observed in FSB positive neurons. Together, these results suggest that within the larger chaperone network, DnaJA2 might be relatively specific to tau pathology in early stages of neurodegeneration. Moreover, this response might be muted or altered in later stages of disease.

\section{Discussion}

Client proteins, such as tau, encounter a network of chaperones and co-chaperones in the cell. In turn, the structural and chemical features of that client are "interpreted" by the chaperones and decisions about the fate of the client (e.g. fold, traffic, degrade) are made. We wanted to understand this molecular recognition process in more detail. Genetic ${ }^{12,55,56}$ and proteomic 17,18 studies have started to identify which chaperones associate with tau and which ones tune its levels. However, we were interested in learning which ones physically 
bind to tau and how tau variants (e.g. mutation, PTMs) impact this molecular recognition. Biochemical and biophysical approaches allowed us to broadly examine tau variants and their direct binding to candidate chaperones. On the other hand, this approach is reductionist in some important ways; for example, chaperones rarely work by themselves and many of them operate in an ATP-rich environment with a full complement of stress responses, associated signaling factors and crowding agents. None of those features are replicated in our studies. Despite this limitation, we uncovered some previously unknown relationships that could have implications for chaperone biology and tauopathies.

\section{Forging new connections between chaperones, tau, and disease-related pathology}

Although DnaJA2 is known to be a co-chaperone of the Hsp70s, it had not previously been linked to tau homeostasis. However, our results suggest that it could be an early marker of tau pathology. Previous transcriptome analyses of brain tissues found a moderate decrease in DnaJA2 mRNA transcripts coincident with age and $\mathrm{AD}^{2}$ however, our results suggest that DnaJA2 protein levels can be highly variable even between individual neurons within the same sample. For example, we found that specific neurons with tau pathology had dramatically higher DnaJA2 levels compared to neighboring cells, which would likely not be clear in whole brain transcriptome studies. Follow-up studies will be aimed at testing the hypothesis that DnaJA2 is an early protective factor that limits tau aggregation. Specifically, it seems possible that deficiencies in either its levels or function may coincide with nucleation sites for pathology.

Other chaperones also warrant further investigation. For example, multiple studies have identified the CLU gene encoding clusterin as a significant risk factor for $\mathrm{AD}^{24}$. Clusterin, a secreted protein, has reported anti-aggregation activities towards $\mathrm{A} \beta$, another amyloidogenic protein implicated in AD. Tau is thought to be secreted from neurons ${ }^{57}$ and elevated tau in the cerebrospinal fluid is a diagnostic marker of $\mathrm{AD}^{58}$. Thus, our findings raise the interesting possibility that clusterin has another protective function to bind and sequester extracellular tau. Another potentially interesting chaperone is the prolyl isomerase, FKBP12. In AD samples, the overall expression of FKBP12 is decreased but a strong co-localization pattern of NFTs with FKBP12 was observed ${ }^{27}$. There is a report that FKBP12 delays aggregation of a short tau peptide containing the PHF6 aggregation motif ${ }^{59}$ however, we found no evidence of anti-aggregation activity in any of the longer tau constructs used in our study. In contrast, our findings suggest that FKBP12 might promote tau aggregation, at least for some acetylated variants.

\section{Do disease-related mutations and PTMs in tau evade chaperone function?}

Tau pathology has been linked to point mutations, truncations and aberrant PTMs. Why do some variants, such as P301L, cause disease? We wondered whether some of these variants might "avoid" the chaperone system, possibly contributing to an imbalance in their own homeostasis. Indeed, we observed a striking reduction in anti-aggregation activity for particular tau variations, such as P301L and D421. Of the 50+ tau mutations linked to tauopathy, P301L is the most common ${ }^{60}$, while accumulation of the D421 fragment is prevalent in $\mathrm{AD}$ patients and is correlated with cognitive decline ${ }^{61}$. Thus, we hypothesize that some tau variants, but likely not all, lose affinity for key surveillance chaperones, such 
as DnaJA2. Over time, this failed recognition might contribute to increased tau levels and its deposition. It is likely that many other factors also contribute, but it is compelling to envision that a failure of surveillance chaperones is one issue.

\section{Online Methods}

\section{Recombinant protein purification}

Methods for purifying individual chaperones are referenced in Supplementary Table 3. Protein purity was confirmed by SDS-PAGE. Modification to published methods are detailed as follows. For tau purification, sodium chloride $(500 \mathrm{mM})$ and a small molecule chaperone, betaine $(10 \mathrm{mM})$, were included in the growth media prior to induction to improve expression and minimize degradation. Expression was induced with $200 \mu \mathrm{M}$ IPTG for $3.5 \mathrm{~h}$ at $30^{\circ} \mathrm{C}$. To purify tau, cells were lysed via a microfluidizer (Microfluidics) followed by boiling of lysate for $20 \mathrm{~min}$. The clarified supernatant containing tau was dialyzed overnight (Buffer A: 20mM MES pH 6.8, $50 \mathrm{mM} \mathrm{NaCl}, 1 \mathrm{mM}$ EGTA, $1 \mathrm{mM} \mathrm{MgCl}$, $2 \mathrm{mM}$ DTT, $0.1 \mathrm{mM}$ PMSF) then purified by cation exchange with a pre-elution step of 4 column volumes of $15 \%$ elution buffer (Buffer $\mathrm{A}$ with $1 \mathrm{M} \mathrm{NaCl}$ ) to remove degradation products. Following a 15-60\% gradient elution, pure tau fractions were pooled and concentrated prior to snap freezing aliquots for storage at $-80 \mathrm{C}$. For K18 tau NMR experiments, tau was purified as described above except that protein was expressed in M9 minimal media containing ${ }^{15} \mathrm{NH}_{4} \mathrm{Cl}(1 \mathrm{~g} / \mathrm{L})$ and $1 \%$ glycerol. Purified ${ }^{15} \mathrm{~N}$-labeled $\mathrm{K} 18$ tau was dialyzed overnight into $20 \mathrm{mM}$ ammonium bicarbonate solution then lyophilized and stored at 4C. Naive Hsp60 containing its mitochondrial import signal was overexpressed in E. coli BL21(DE3) cells induced with $0.25 \mathrm{M}$ IPTG for $4 \mathrm{~h}$ at 37C. Cells were re-suspended in $50 \mathrm{mM}$ Tris- $\mathrm{HCl} \mathrm{pH} \mathrm{8,10} \mathrm{mM} \mathrm{imidazole,} 500 \mathrm{mM} \mathrm{NaCl}$ and lysed with a microfluidizer. The cleared lysate was applied Ni-NTA resin (Qiagen) and eluted with buffer containing 300 $\mathrm{mM}$ imidazole. The His tag was cleaved during overnight dialysis in $50 \mathrm{mM}$ Tris $\mathrm{pH} 7.5,1$ mM DTT at 4C. Hsp60 was further purified using a Superdex 200 16/600 column (GE Healthcare) equilibrated with $50 \mathrm{mM}$ Tris- $\mathrm{HCl}$ pH 7.5, $150 \mathrm{mM} \mathrm{NaCl}$. Hsp60 14-mers were concentrated, snap frozen in liquid nitrogen and stored at $-80 \mathrm{C}$.

Wild-type Pin1 was cloned into the pMCSG10 vector and transfected into BL21 (DE3) cells. Cultures were grown at $37^{\circ} \mathrm{C}$ to an approximate OD of 2 then expressed with $1 \mathrm{mM}$ IPTG overnight at room temperature. Cell pellets were collected and lysed (100mM Tris, 200mM $\mathrm{NaCl}, 10 \%$ Glycerol, $1 \mathrm{mM}$ reduced GSH, pH 8) then loaded on to a GSTrap 4B column. After purification, TEV protease was added overnight. Cleaved Pin1 was concentrated and purified by size-exclusion chromatography on a Superdex 75 column (10mM HEPES, $10 \mathrm{mM} \mathrm{NaCl}, 10 \%$ Glycerol, 1mM DTT, pH 7.5). S. cerevisiae Hsp104/pProEX-HTb was overexpressed with a TEV protease-cleavable $\mathrm{N}$-terminal His $6_{6}$ tag in E. coli BL21 (DE3)RIL (Agilent) by inducing cells with $0.5 \mathrm{mM}$ IPTG for $6 \mathrm{~h}$ at $25^{\circ} \mathrm{C}$. Cells were resuspended in $25 \mathrm{mM}$ Tris- $\mathrm{HCl} \mathrm{pH} \mathrm{7.5,} 300 \mathrm{mM} \mathrm{NaCl}, 5 \%$ glycerol, $30 \mathrm{mM}$ imidazole, and $5 \mathrm{mM} \beta$ mercaptoethanol, and lysed using a microfluidizer. The cleared lysate was applied to a $\mathrm{Ni}$ Sepharose FF column (GE Healthcare) and proteins were eluted using a linear gradient of 30 $\mathrm{mM}$ to $500 \mathrm{mM}$ imidazole. The $\mathrm{His}_{6}$-tag was cleaved with $\mathrm{His}_{6}$-TEV protease while dialyzing the protein sample in $50 \mathrm{mM}$ Tris- $\mathrm{HCl} \mathrm{pH} 7.5,50 \mathrm{mM} \mathrm{NaCl}, 5 \%$ glycerol, and 1 
mM DTT overnight at $4^{\circ} \mathrm{C}$. After removing the liberated His-tag and $\mathrm{His}_{6}-\mathrm{TEV}$ protease by passing over a Ni-Sepharose FF column, Hsp104 was purified by anion exchange chromatography and eluted using a linear gradient of $50 \mathrm{mM}$ to $1000 \mathrm{mM}$ of $\mathrm{NaCl}$. Hsp104 was further purified by size-exclusion chromatography using a Superdex 200 HiLoad 16/60 prep-grade column (GE Healthcare) pre-equilibrated in $25 \mathrm{mM}$ Tris- $\mathrm{HCl} \mathrm{pH} \mathrm{7.5,150} \mathrm{mM}$ $\mathrm{NaCl}, 5 \%$ glycerol and $1 \mathrm{mM}$ DTT. Hsp104 hexamers were concentrated, snap-frozen in liquid nitrogen and stored at $-80{ }^{\circ} \mathrm{C}$.

\section{In vitro tau aggregation}

All proteins in assay were dialyzed overnight at $4{ }^{\circ} \mathrm{C}$ into assay buffer (Dulbecco's PBS $\mathrm{pH} 7.4,2 \mathrm{mM} \mathrm{MgCl} 2,1 \mathrm{mM}$ DTT). Tau $(10 \mu \mathrm{M})$ was pre-incubated in the presence or absence of chaperones $(2.5 \mu \mathrm{M}-20 \mu \mathrm{M})$ for $30 \mathrm{~min}$ at $37{ }^{\circ} \mathrm{C}$. Thioflavin $\mathrm{T}$ (Sigma) at a final concentration of $10 \mu \mathrm{M}$ was added and aggregation was induced by the addition of a freshly prepared heparin sodium salt solution (Santa Cruz) at a final concentration of $44 \mathrm{ug} / \mathrm{ml}$. For non-induced controls, assay buffer was added in place of heparin solution. In assays using arachadonic acid as an aggregation inducer, heparin was replaced with arachadonic acid $(150 \mu \mathrm{M})$ in 5\% ethanol and non-induced controls contained 5\% ethanol. Aggregation reactions were carried out at $37^{\circ} \mathrm{C}$ with continuous shaking and monitored via Thioflavin $\mathrm{T}$ fluorescence (excitation $=444 \mathrm{~nm}$, emission $=485 \mathrm{~nm}$, cutoff $=480 \mathrm{~nm}$ ) in a Spectramax M5 microplate reader (Molecular Devices). Readings were taken every $5 \mathrm{~min}$ for a minimum of $24 \mathrm{~h}$. Note that for experiments with $\mathrm{Hsp} 72_{\mathrm{SBD}}$, assays were performed at $30^{\circ} \mathrm{C}$, well below its observed protein melting point $\left(42^{\circ} \mathrm{C}\right)$. Chaperones with poor solubility in standard screen conditions as indicated in the text were assayed in reactions with the following modifications: the tau concentration was reduced to $7.5 \mathrm{uM}$ and the aggregation reaction was carried out in 96 well plates with total volumes of 200 ul per well.

For data processing, baseline curves of non-induced controls ( 3 replicates) were subtracted from induced samples (3 replicates). Baseline subtracted aggregation curves were analyzed in Grace plotting program (http://plasma-gate.weizmann.ac.il/Grace/) and fit to the Goempertz function ${ }^{62,63}$ :

$$
y=A e^{-e^{\left(t-t_{i}\right) / b}}
$$

where $y=$ the fluorescence at time $t, A$ is the amplitude of curve, $t_{i}$ is the inflection point, $t_{i}-b$ $=$ lag time, and $b=1 / k_{a p p}$ where $k_{a p p}$ is the apparent elongation rate constant. Amplitude values are represented relative to $0 \mathrm{~N} 4 \mathrm{R}$ tau ${ }^{\mathrm{WT}}$ controls for each experiment to adjust for day to day fluctuations in absolute ThT signal intensity. Curves with final amplitude values $<15 \%$ of the internal tau alone controls were classified as non-aggregating. For the primary aggregation screen, each experiment contained multiple reference controls including $\mathrm{Hsc70}$ NBD and Hsc70 (at each concentrated tested) and 0N4R tau ${ }^{\mathrm{WT}}$ tau $(10 \mu \mathrm{M})$. The effect of each chaperone on aggregation kinetics is normalized to the values for the internal Hsc70 ${ }_{\mathrm{NBD}}$ and $\mathrm{Hsc} 70$ controls. Heat maps for chaperone effects on aggregation kinetics were generated in $\mathrm{R}^{64}$. For experiments involving chaperone addition after lag phase period, 
the aggregation curve for $4 \mathrm{~h}$ immediately following addition was fit with linear regression to calculate the slope.

\section{In vitro tau seeding reaction}

Tau $(10 \mathrm{uM})$ was aggregated in $1.7 \mathrm{ml}$ microcentrifuge tubes under conditions similar to the aggregation screen except that ThT was omitted. When indicated $10 \mathrm{uM}$ chaperone was also present. Unfractionated aggregate mixtures were quantified by gel electrophoresis then added at a final concentration of ( $30 \mathrm{nM}$ tau) to seed fresh tau monomer solutions (10 uM tau, Dulbecco's PBS pH7.4, 2 mM MgCl 2,1 mM DTT, $10 \mathrm{uM} \mathrm{ThT,} 44 \mathrm{ug} / \mathrm{ml}$ heparin. The aggregation reaction was monitored and analyzed as described in the previous section (In vitro tau aggregation).

\section{Tau peptide microarray}

Experimental design and procedure for the tau peptide microarray has been previously described ${ }^{17}$. Briefly, the microarrays were printed on single microscope slides in triplicate with peptides covalently immobilized at the $\mathrm{N}$-terminus (Jenrin Peptide Technologies, Berlin, Germany). Peptide binding was tested per manufacturer's protocol using $10 \mu \mathrm{M}$ of His6x-tagged protein in binding buffer (25 mM HEPES pH 7.2, $150 \mathrm{mM} \mathrm{NaCl}, 20 \mathrm{mM} \mathrm{KCl}$, $5 \mathrm{mM} \mathrm{MgCl}_{2}, 0.01 \%$ Tween20). Binding was detected with a 1:1,000 dilution of HiLyte555 anti-His6x antibody (Anaspec, Fremont, CA) in TBS-T with 1\% bovine serum albumin (Sigma) and scanning the microarrays using a GenePix 4100A Microarray Scanner (Molecular Devices, Sunnyvale, CA) at an excitation wavelength of $532 \mathrm{~nm}$. Empty spots were used as negative controls. Binding was defined as $>2$ consecutive peptides with fluorescence signals greater than 1 standard deviation above the mean in replicate experiments. Peptides that bound to the anti-His6X antibody alone or to an Hsc70 construct lacking its substrate-binding domain $\left(\mathrm{Hsc} 70_{\mathrm{NBD}}\right)$ were identified as false positives and excluded from further analysis. Positive sites were restricted to those composed of two or more consecutive binding peptides. The one exception was peptide (aa 5-29), which was included because the peptide preceding it was a false positive.

\section{NMR Spectroscopy}

For K18 tau-chaperone binding studies, all proteins were dialyzed overnight into NMR buffer. NMR buffer used in experiments was $50 \mathrm{mM}$ sodium phosphate $\mathrm{pH} 6.8,10 \mathrm{mM}$ $\mathrm{NaCl}, 1 \mathrm{mM}$ TCEP, $10 \%$ (v/v) $\mathrm{D}_{2} \mathrm{O}$ ) or alternatively, $25 \mathrm{mM}$ HEPES $\mathrm{pH}$ 7.4, $10 \mathrm{mM} \mathrm{KCl}, 5$ $\mathrm{mM} \mathrm{MgCl} 2,1 \mathrm{mM}$ TCEP, $10 \%$ (v/v) D2O). Prepared samples contained $50 \mu \mathrm{M}^{15} \mathrm{~N}$-labeled Tau and 50 or $100 \mu \mathrm{M}$ unlabeled chaperone in NMR buffer. Two-dimensional (2D) ${ }^{1} \mathrm{H}_{-}{ }^{15} \mathrm{~N}$ hetero- nuclear single quantum coherence (HSQC) spectra were required at $10^{\circ} \mathrm{C}$ on a Bruker Avance AV800 spectrometer equipped with a cryoprobe. 16 scans were acquired per t1 value and spectral widths of $2100 \mathrm{~Hz}$ and $10416 \mathrm{~Hz}$ were used in the ${ }^{1} \mathrm{H}$ and ${ }^{15} \mathrm{~N}$ dimensions, respectively. Spectra were processed using rNMR and Sparky based on deposited tau assignments (Barre 2013, BioMagResBank accession number 19253). Signal intensity ratios were calculated using Prism. For $2 \mathrm{~N} 4 \mathrm{R}$ tau interaction studies, ${ }^{1} \mathrm{H}_{-}{ }^{15} \mathrm{~N}$ correlation spectra were acquired on $30 \mu \mathrm{M}{ }^{15} \mathrm{~N}$-labeled tau and up to 10 equivalents of unlabeled chaperone at $5^{\circ} \mathrm{C}$ on Bruker Avance III $800 \mathrm{MHz}$ and $900 \mathrm{MHz}$ spectrometers (both equipped with TCI cryoprobes). $25 \mathrm{mM}$ HEPES, $10 \mathrm{mM} \mathrm{KCl}, 5 \mathrm{mM} \mathrm{MgCl}_{2}, 1 \mathrm{mM}$ 
TCEP, 10\% (v/v) D2O), pH 7.4 was used as experimental buffer for the ATP-dependent chaperones (DJA2, DJB2, DJB4, Hsp72 and Hsp90ß), while $50 \mathrm{mM}$ sodium phosphate, 10 $\mathrm{mM} \mathrm{NaCl}, 1 \mathrm{mM}$ DTT, $10 \%$ (v/v) $\mathrm{D}_{2} \mathrm{O}$ ), $\mathrm{pH} 6.8$ was used for Hsp27 and AB-crystallin. Spectra were processed in Topspin and analyzed in Sparky.

\section{Tau cellular seeding assay}

The HEK293 cell line stably expressing the microtubule-binding repeat region of tau fused to yellow fluorescent protein (clone 1) was kindly provided by Marc Diamond ${ }^{54}$. The cell line stock tested negative for mycoplasma. Cells were plated at a density of 2000 cells/well in 384 well clear black plates (Greiner Bio-One) and immediately treated with unfractionated tau fibril preparations ( $0-1 \mu \mathrm{g}$ tau/well). For experiments where lipofectamine 2000 (Thermo Fisher Scientific) used to introduce fibrils, a ratio of $1 \mu \mathrm{g}$ tau protein: $0.3 \mathrm{uL}$ lipofectamine 2000 was used. Following a $48 \mathrm{~h}$ treatment, nuclei were stained in live cells with $0.012 \mu \mathrm{g} /$ well of Hoescht 33342 (Thermo Fisher Scientific) for $1 \mathrm{~h}$. Images for DAPI and FITC channels from 3 regions per well were captured using an InCell 6000 (GE HealthCare). The images were processed using InCell Developer software (GE HealthCare) with an algorithm developed to identify live cells with intracellular aggregates larger than $0.89 \mu \mathrm{m}^{2}$. Greater than 600 cells per well, assayed in triplicate, was analyzed in 3 independent experiments.

\section{Fibril sedimentation}

Tau aggregation samples were centrifuged at $100,000 \mathrm{~g}$ for $1 \mathrm{~h}$ at $4{ }^{\circ} \mathrm{C}$. Supernatants were collected and pellets were suspended in aggregation assay buffer overnight at room temperature with shaking. SDS sample buffer (1X final) was added to equal fractions of each reaction and loaded on 4-15\% SDS-PAGE gels. A set of standards of known tau protein amounts was present on each gel. Proteins were visualized with coomassie blue and images of stained gels were used to quantify the bands with Image lab software (Bio-Rad).

\section{Human Tissue Samples}

7 formalin-fixed brain tissue samples were obtained from neuropathologically confirmed cases of Alzheimer's disease (Braak stage V-VI), mild cognitive impairment (Braak stage II), or non-demented aged control. Medial frontal cortex and inferior temporal cortex regions were utilized for this study.

\section{Immunohistochemistry and Confocal imaging}

Formalin-fixed samples were embedded in paraffin using standard procedures. Sections (8 $\mu \mathrm{m})$ were cut, photobleached using a multispectral LED lamp, deparaffinized, and then processed for immunohistochemistry. Sections stained with antibodies were pretreated by incubation in heated $0.01 \mathrm{M}$ sodium citrate buffer as an epitope retrieval step. Following blocking with $10 \%$ normal goat serum, sections were incubated with primary antibody overnight at $4{ }^{\circ} \mathrm{C}$ (see Supp. Table 4 for antibody specfications). Primary antibody detection was performed using goat secondary antibodies with conjugated AlexaFluor 488 or AlexaFluor 647 (A-21245, Life Technologies). All immunohistochemistry-stained sections were subsequently stained with $2.5 \mu \mathrm{M}$ fluoro-styrylbenzene (FSB; Congo red derivative) 
(Santa Cruz) in $1 \times$ PBS and propidium iodide (Sigma) washed before mounting. Samples were visualized using a $40 \times$ water-immersion lens (1.1 NA) or $63 x$ oil-immersion (1.4 NA) in sequential scan mode on a Leica SP8 confocal microscope equipped with $H y D$ detectors. 8 -bit image z-stacks ( 1 - $\mu \mathrm{m}$ steps) were collected at $1024 \times 1024$ or $2048 \times 2048$ pixel resolution. Images were processed using NIH ImageJ.

\section{Isothermal calorimetry (ITC)}

Chaperones were dialyzed into ITC buffer overnight (40 mM HEPES pH7.4, $150 \mathrm{mM} \mathrm{NaCl}$. Protein concentrations were determined via BCA assay (Pierce). The experiment was carried out at $25^{\circ} \mathrm{C}$ on a MicroCal VP-ITC where heparin at $200 \mu \mathrm{M}$ or $1 \mathrm{mM}$ (based on an average MW of 9500) was titrated into a $100 \mu \mathrm{M}$ cell solution of Hsc70 or DnaJA2. Calorimetric parameters were calculated using Origin ${ }^{\circledR} 7.0$ software and fit with a one-site binding model.

\section{Dot blot}

Purified recombinant chaperones (1.5 ug or 0.75 ug total) were spotted on nitrocellulose, stained with Ponceau $\mathrm{S}$ to verify transfer, then processed by immunoblot. The membrane was blocked in $4 \%$ milk solution then incubated with DnaJA2 antibody $(1: 20,000)$ at $4{ }^{\circ} \mathrm{C}$, overnight. The membrane was incubated with goat anti-mouse HRP $(1: 40,000$, Cell Signaling Technology) for 45 min prior to chemiluminescence signal detection with ECL Select (Amersham) by a Chemidoc Touch gel imaging system (Bio-Rad).

\section{Data Availability Statement}

The datasets generated and analyzed in the current study are available from the corresponding author upon reasonable request.

\section{Supplementary Material}

Refer to Web version on PubMed Central for supplementary material.

\section{Acknowledgments}

Funding:

This work was funded by grants to J.E.G. from the Tau Consortium, BrightFocus Foundation and the NIH (NS059690) and to F.T.F.T from the NIH (GM104980 and GM111084). The funders had no role in study design, data collection and interpretation, or the decision to submit the work for publication.

We thank Erin Poss and Kartika Widjaja for technical support, Susmitha Ambadipudi for NMR analysis and Jan Stoehr for advice on ThT assays. We are grateful to the Bardwell group (U. Michigan) for supplying chaperone proteins, Marc Diamond (Tau consortium) for providing Clone 1 cells. Human autopsied brain tissue was received from William W. Seeley and the UCSF Neurodegenerative Disease Brain Bank, which is supported by the NIH (AG023501 and AG19724 to W.W.S.), the Tau Consortium, and the Consortium for Frontotemporal Dementia Research.

\section{References}

1. Kim YE, Hipp MS, Bracher A, Hayer-Hartl M, Hartl FU. Molecular chaperone functions in protein folding and proteostasis. Annu Rev Biochem. 2013; 82:323-55. [PubMed: 23746257] 
2. Brehme M, et al. A Chaperome Subnetwork Safeguards Proteostasis in Aging and Neurodegenerative Disease. Cell Rep. 2014; 9:1135-50. [PubMed: 25437566]

3. Taipale M, et al. A quantitative chaperone interaction network reveals the architecture of cellular protein homeostasis pathways. Cell. 2014; 158:434-448. [PubMed: 25036637]

4. Rodina A, et al. The epichaperome is an integrated chaperome network that facilitates tumour survival. Nature. 2016; 538:397-401. [PubMed: 27706135]

5. Mu TW, et al. Chemical and Biological Approaches Synergize to Ameliorate Protein-Folding Diseases. Cell. 2008; 134:769-781. [PubMed: 18775310]

6. Morimoto RI. The heat shock response: Systems biology of proteotoxic stress in aging and disease. Cold Spring Harb Symp Quant Biol. 2011; 76:91-99. [PubMed: 22371371]

7. Walther DM, et al. Widespread Proteome Remodeling and Aggregation in Aging C. elegans. Cell. 2015; 161:919-932. [PubMed: 25957690]

8. Soto C. Unfolding the role of protein misfolding in neurodegenerative diseases. Nat Rev Neurosci. 2003; 4:49-60. [PubMed: 12511861]

9. Hsu AL, Murphy CT, Kenyon C. Regulation of Aging and Age-Related Disease by DAF-16 and Heat-Shock Factor. Science. 2003; 300:1142-1145. [PubMed: 12750521]

10. Cohen E, Bieschke J, Perciavalle RM, Kelly JW, Dillin A. Opposing activities protect against ageonset proteotoxicity. Science. 2006; 313:1604-10. [PubMed: 16902091]

11. Krobitsch S, Lindquist S. Aggregation of huntingtin in yeast varies with the length of the polyglutamine expansion and the expression of chaperone proteins. Proc Natl Acad Sci. 2000; 97:1589-1594. [PubMed: 10677504]

12. Abisambra JF, et al. Phosphorylation Dynamics Regulate Hsp27-Mediated Rescue of Neuronal Plasticity Deficits in Tau Transgenic Mice. 2010; 30:15374-15382.

13. Jinwal UK, et al. Chemical manipulation of hsp70 ATPase activity regulates tau stability. J Neurosci. 2009; 29:12079-88. [PubMed: 19793966]

14. Kakkar V, Kuiper EFE, Pandey A, Braakman I, Kampinga HH. Versatile members of the DNAJ family show Hsp70 dependent anti-aggregation activity on RING1 mutant parkin C289G. Sci Rep. 2016; 6:34830. [PubMed: 27713507]

15. Spillantini MG, Goedert M. Tau pathology and neurodegeneration. Lancet Neurol. 2013; 12:609622. [PubMed: 23684085]

16. Young ZT, Mok SA, Gestwicki JE. Therapeutic Strategies for Restoring Tau Homeostasis. Cold Spring Harb Perspect Med. 2017; doi: 10.1101/cshperspect.a024612

17. Thompson AD, et al. Analysis of the tau-associated proteome reveals that exchange of Hsp70 for Hsp90 is involved in tau degradation. ACS Chem Biol. 2012; 7:1677-86. [PubMed: 22769591]

18. Gunawardana CG, et al. The human tau interactome: binding to the ribonucleoproteome, and impaired binding of the P301L mutant to chaperones and the proteasome. Mol Cell Proteomics. 2015; 14:3000-3014. [PubMed: 26269332]

19. Jinwal UK, et al. The Hsp90 Cochaperone, FKBP51, Increases Tau Stability and Polymerizes Microtubules. 2010; 30:591-599.

20. Voss K, Combs B, Patterson KR, Binder LI, Gamblin TC. Hsp70 alters tau function and aggregation in an isoform specific manner. Biochemistry. 2012; 51:888-98. [PubMed: 22236337]

21. von Bergen M, et al. Mutations of tau protein in frontotemporal dementia promote aggregation of paired helical filaments by enhancing local beta-structure. J Biol Chem. 2001; 276:48165-74. [PubMed: 11606569]

22. von Bergen M, et al. Assembly of tau protein into Alzheimer paired helical filaments depends on a local sequence motif ((306)VQIVYK(311)) forming beta structure. Proc Natl Acad Sci U S A. 2000; 97:5129-5134. [PubMed: 10805776]

23. Li W, Lee VMY. Characterization of two VQIXXK motifs for tau fibrillization in vitro. Biochemistry. 2006; 45:15692-701. [PubMed: 17176091]

24. Van Cauwenberghe C, Van Broeckhoven C, Sleegers K. The genetic landscape of Alzheimer disease: clinical implications and perspectives. Genet Med. 2016; 18:421-430. [PubMed: 26312828] 
25. Shinohara H, Inaguma Y, Goto S, Inagaki T, Kato K. aB crystallin and HSP28 are enhanced in the cerebral cortex of patients with Alzheimer's disease. J Neurol Sci. 1993; 119:203-208. [PubMed: 8277336]

26. Lim J, et al. Pin1 has opposite effects on wild-type and P301L tau stability and tauopathy. 2008; 118:1877-1889.

27. Sugata H, et al. A peptidyl-prolyl isomerase, FKBP12, accumulates in Alzheimer neurofibrillary tangles. Neurosci Lett. 2009; 459:96-99. [PubMed: 19414059]

28. Hansen J, et al. Decreased expression of the mitochondrial matrix proteases Lon and ClpP in cells from a patient with hereditary spastic paraplegia (SPG13). Neuroscience. 2008; 153:474-482. [PubMed: 18378094]

29. Howarth JL, Glover CPJ, Uney JB. HSP70 interacting protein prevents the accumulation of inclusions in polyglutamine disease. J Neurochem. 2009; 108:945-951. [PubMed: 19183265]

30. Lee J, et al. Heat shock protein (Hsp) 70 is an activator of the Hsp104 motor. Proc Natl Acad Sci. 2013; 110:8513-8518. [PubMed: 23650362]

31. Quan S, et al. Genetic selection designed to stabilize proteins uncovers a chaperone called Spy. Nat Struct Mol Biol. 2011; 18:262-269. [PubMed: 21317898]

32. Lennon CW, et al. Folding Optimization in Vivo Uncovers New Chaperones. J Mol Biol. 2015; 427:2983-2994. [PubMed: 26003922]

33. Yerbury JJ, et al. The extracellular chaperone clusterin influences amyloid formation and toxicity by interacting with prefibrillar structures. FASEB J. 2007; 21:2312-22. [PubMed: 17412999]

34. Arimon M, Grimminger V, Sanz F, Lashuel HA. Hsp104 Targets Multiple Intermediates on the Amyloid Pathway and Suppresses the Seeding Capacity of A?? Fibrils and Protofibrils. J Mol Biol. 2008; 384:1157-1173. [PubMed: 18851977]

35. Glover JR, Lindquist S. Hsp104, Hsp70, and Hsp40: A novel chaperone system that rescues previously aggregated proteins. Cell. 1998; 94:73-82. [PubMed: 9674429]

36. Niblock M, Gallo J. Tau alternative splicing in familial and sporadic tauopathies. Biochem Soc Trans. 2012; 40:677-680. [PubMed: 22817715]

37. Jinwal UK, et al. Imbalance of Hsp70 family variants fosters tau accumulation. FASEB J. 2013; 27:1450-9. [PubMed: 23271055]

38. Young ZT, et al. Stabilizing the Hsp70-Tau Complex Promotes Turnover in Models of Tauopathy. Cell Chem Biol. 2016; 23:992-1001. [PubMed: 27499529]

39. Arosio P, Knowles TPJ, Linse S. On the lag phase in amyloid fibril formation. Phys Chem Chem Phys. 2015; 17:7606-7618. [PubMed: 25719972]

40. Arosio P, Knowles TPJ, Linse S. On the lag phase in amyloid fibril formation. Phys Chem Chem Phys. 2015; 17:7606-7618. [PubMed: 25719972]

41. Arosio P, et al. Kinetic analysis reveals the diversity of microscopic mechanisms through which molecular chaperones suppress amyloid formation. Nat Commun. 2016; 7:10948. [PubMed: 27009901]

42. Gao X, et al. Human Hsp70 Disaggregase Reverses Parkinson's-Linked a-Synuclein Amyloid Fibrils. Mol Cell. 2015; 59:781-793. [PubMed: 26300264]

43. Verba KA, et al. Atomic structure of Hsp90-Cdc37-Cdk4 reveals that Hsp90 traps and stabilizes an unfolded kinase. Science. 2016; 352:1542-1547. [PubMed: 27339980]

44. Kirschke E, Goswami D, Southworth D, Griffin PR, Agard DA. Glucocorticoid receptor function regulated by coordinated action of the Hsp90 and Hsp70 chaperone cycles. Cell. 2014; 157:16851697. [PubMed: 24949977]

45. Karagöz GE, et al. Hsp90-Tau Complex Reveals Molecular Basis for Specificity in Chaperone Action. Cell. 2014; 156:963-974. [PubMed: 24581495]

46. Cheng G, Basha E, Wysocki VH, Vierling E. Insights into small heat shock protein and substrate structure during chaperone action derived from hydrogen/deuterium exchange and mass spectrometry. J Biol Chem. 2008; 283:26634-26642. [PubMed: 18621732]

47. Wang JZ, Xia YY, Grundke-Iqbal I, Iqbal K. Abnormal hyperphosphorylation of tau: Sites, regulation, and molecular mechanism of neurofibrillary degeneration. Adv Alzheimer's Dis. 2012; 3:123-139. 
48. Nakamura K, et al. Proline isomer-specific antibodies reveal the early pathogenic tau conformation in Alzheimer's disease. Cell. 2012; 149:232-44. [PubMed: 22464332]

49. Combs B, Hamel C, Kanaan NM. Pathological conformations involving the amino terminus of tau occur early in Alzheimer's disease and are differentially detected by monoclonal antibodies. Neurobiol Dis. 2016; 94:18-31. [PubMed: 27260838]

50. Kfoury N, Holmes BB, Jiang H, Holtzman DM, Diamond MI. Trans-cellular propagation of Tau aggregation by fibrillar species. J Biol Chem. 2012; 287:19440-51. [PubMed: 22461630]

51. Clavaguera F, et al. Transmission and spreading of tauopathy in transgenic mouse brain. Nat Cell Biol. 2009; 11:909-913. [PubMed: 19503072]

52. Prusiner SB. Cell biology. A Unifying Role for Prions in Neurodegenerative Diseases. Science. 2012; 336:1511-1513. [PubMed: 22723400]

53. Mirbaha H, Holmes BB, Sanders DW, Bieschke J, Diamond MI. Tau Trimers Are the Minimal Propagation Unit Spontaneously Internalized to Seed Intracellular. 2015; 290:14893-14903.

54. Sanders DW, et al. Article Distinct Tau Prion Strains Propagate in Cells and Mice and Define Different Tauopathies. Neuron. 2014; 82:1271-1288. [PubMed: 24857020]

55. Fontaine SN, et al. Isoform Selective Genetic Inhibition of Constitutive Cytosolic Hsp70 Activity Promotes Client Tau Degradation Using an Altered Co-chaperone Complement. J Biol Chem. 2015; 290:13115-27. [PubMed: 25864199]

56. Dickey CA, et al. Deletion of the ubiquitin ligase CHIP leads to the accumulation, but not the aggregation, of both endogenous phospho- and caspase-3-cleaved tau species. J Neurosci. 2006; 26:6985-96. [PubMed: 16807328]

57. Gendreau KL, Hall GF. Tangles, Toxicity, and Tau Secretion in AD - New Approaches to a Vexing Problem. Front Neurol. 2013; 4:160. [PubMed: 24151487]

58. Pooler AM, et al. Propagation of tau pathology in Alzheimer's disease: Identification of novel therapeutic targets. Alzheimer's Res Ther. 2013; 5:49. [PubMed: 24152385]

59. Ikura T, Ito N. Peptidyl-prolyl isomerase activity of FK506 binding protein 12 prevents tau peptide from aggregating. Protein Eng Des Sel. 2013; 26:539-46. [PubMed: 23832849]

60. Ghetti B, et al. Invited review: Frontotemporal dementia caused by microtubule-associated protein tau gene (MAPT) mutations: a chameleon for neuropathology and neuroimaging. Neuropathol Appl Neurobiol. 2015; 41:24-46. [PubMed: 25556536]

61. Rissman RA, et al. Caspase-cleavage of tau is an early event in Alzheimer disease tangle pathology. J Clin Invest. 2004; 114:121-130. [PubMed: 15232619]

62. Moore CL, et al. Secondary nucleating sequences affect kinetics and thermodynamics of tau aggregation. Biochemistry. 2011; 50:10876-86. [PubMed: 22085312]

63. Winsor CP. The Gompertz Curve as a Growth Curve. Proc Natl Acad Sci. 1932; 18:1-8. [PubMed: 16577417]

64. R-Core-Team. R: A language and environment for statistical computing. R Foundation for Statistical Computing; 2013.

65. Coppola G, et al. Evidence for a role of the rare p.A152T variant in MAPT in increasing the risk for FTD-spectrum and Alzheimer's diseases. Hum Mol Genet. 2012; 21:3500-12. [PubMed: 22556362]

66. Augustinack J, Schneider A, Mandelkow EM, Hyman B. Specific tau phosphorylation sites correlate with severity of neuronal cytopathology in Alzheimer's disease. Acta Neuropathol. 2002; 103:26-35. [PubMed: 11837744]

67. Min SW, et al. Critical role of acetylation in tau-mediated neurodegeneration and cognitive deficits. Nat Med. 2015; 21:1154-62. [PubMed: 26390242]

68. Cohen TJ, et al. The acetylation of tau inhibits its function and promotes pathological tau aggregation. Nat Commun. 2011; 2:252-259. [PubMed: 21427723]

69. Corsetti V, et al. Identification of a caspase-derived N-terminal tau fragment in cellular and animal Alzheimer's disease models. Mol Cell Neurosci. 2008; 38:381-92. [PubMed: 18511295]

70. Barghorn S, Biernat J, Mandelkow E. Purification of Recombinant Tau Protein and Preparation of Alzheimer-Paired Helical Filaments In Vitro. Methods Mol Biol. 2005; 299:35-51. [PubMed: 15980594] 
71. Mukrasch MD, et al. Sites of tau important for aggregation populate \{beta\}-structure and bind to microtubules and polyanions. J Biol Chem. 2005; 280:24978-86. [PubMed: 15855160]

72. Thompson AD, Bernard SM, Skiniotis G, Gestwicki JE. Visualization and functional analysis of the oligomeric states of Escherichia coli heat shock protein 70 (Hsp70/DnaK). Cell Stress Chaperones. 2012; 17:313-27. [PubMed: 22076723]

73. Chang L, et al. Chemical screens against a reconstituted multiprotein complex: Myricetin blocks DnaJ regulation of DnaK through an allosteric mechanism. Chem Biol. 2011; 18:210-221. [PubMed: 21338918]

74. Rauch JN, Gestwicki JE. Binding of human nucleotide exchange factors to heat shock protein 70 (Hsp70) generates functionally distinct complexes in vitro. J Biol Chem. 2014; 289:1402-14. [PubMed: 24318877]

75. Rauch JN, et al. BAG3 Is a Modular, Scaffolding Protein that physically Links Heat Shock Protein 70 (Hsp70) to the Small Heat Shock Proteins. J Mol Biol. 2017; 429:128-141. [PubMed: 27884606]

76. Makley LN, et al. Pharmacological chaperone for a-crystallin partially restores transparency in cataract models. Science. 2015; 350:674-677. [PubMed: 26542570]

77. Dunyak BM, Nakamura RL, Frankel AD, Gestwicki JE. Selective Targeting of Cells via Bispecific Molecules That Exploit Coexpression of Two Intracellular Proteins. ACS Chem Biol. 2015; 10:2441-2447. [PubMed: 26322864]

78. Assimon VA, Southworth DR, Gestwicki JE. Specific Binding of Tetratricopeptide Repeat Proteins to Heat Shock Protein 70 (Hsp70) and Heat Shock Protein 90 (Hsp90) Is Regulated by Affinity and Phosphorylation. Biochemistry. 2015; 54:7120-7131. [PubMed: 26565746]

79. Wilson MR, Easterbrook-Smith SB. Clusterin binds by a multivalent mechanism to the Fc and Fab regions of IgG. Biochim Biophys Acta (BBA)/Protein Struct Mol. 1992; 1159:319-326.

80. Morishima Y, et al. Heme-dependent activation of neuronal nitric oxide synthase by cytosol is due to an hsp70-dependent, thioredoxin-mediated thiol - Disulfide interchange in the heme/substrate binding cleft. Biochemistry. 2011; 50:7146-7156. [PubMed: 21755988] 


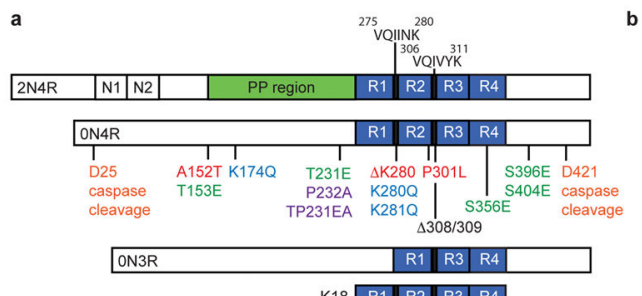

b

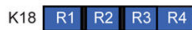
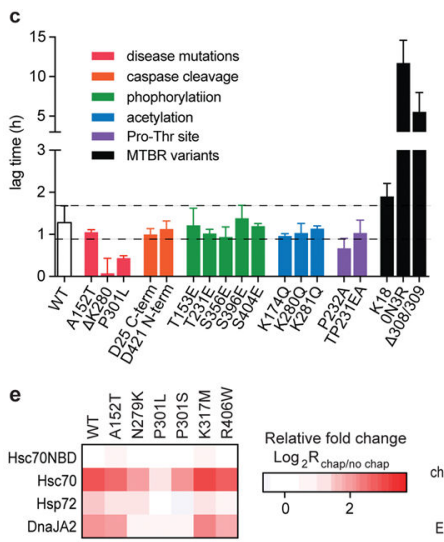

Relative fold change

d
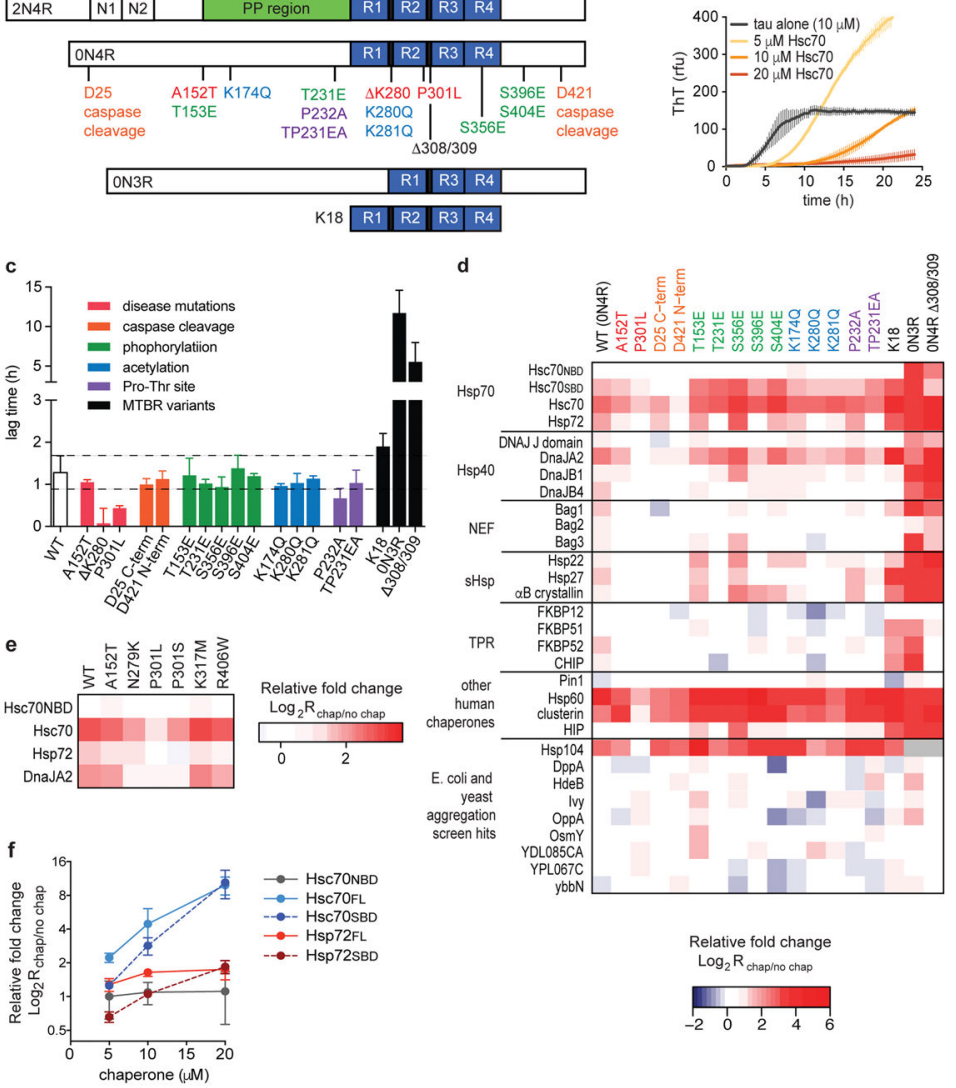

Figure 1. In vitro tau aggregation screen identifies chaperones that inhibit or promote tau aggregation

a) Schematic of the longest splice isoform of tau (2N4R) is shown (N1 and N2 $=\mathrm{N}$-terminal inserts; $\mathrm{PP}$ region = polyproline region; $\mathrm{R} 1$ through $\mathrm{R} 4=\mathrm{MBRs}$ ) and the location of aggregation motifs (VQIINK and VQIVYK) are indicated. Below are schematics of tau variants assayed in screen $(0 \mathrm{~N} 4 \mathrm{R}, 0 \mathrm{~N} 3 \mathrm{R}$ and K18). Location of variants referenced on the ON4R tau construct: disease-associated mutations (red) and PTMs (orange: caspase cleavage site, green: phosphorylation site, blue: acetylation site, purple: proline isomerization sites). b) Sample result from the ThT-based tau aggregation assay. Aggregation of recombinant ON4R tau ${ }^{\mathrm{WT}}(10 \mu \mathrm{M})$ induced by heparin in the presence of Hsc70. Representative data from one of three independent experiments plotted (mean \pm SEM, triplicates). c) Lag time values for tau variants in the ThT assay. Aggregation of tau variants $(10 \mu \mathrm{M})$ was induced with heparin and calculated lag time extracted from ThT curves was plotted (mean $\pm \mathrm{SD}$, triplicates). d) Heatmap depicting the effect of individual chaperones on the lag time parameter of aggregation curves. For each chaperone-tau combination, the $\log _{2}$ fold change in lag time when aggregated in the absence or presence of an equimolar concentration of chaperone $(10 \mu \mathrm{M})$ was plotted. The legend key indicates the effect size and direction. Grey = not tested. e) Heatmap depicting that a subset of disease-associated mutation are resistant to chaperone protection from aggregation. Data plotted as described in d). f) Differences between $\mathrm{Hsc70}$ and $\mathrm{Hsp} 72$ anti-aggregation effects attributed to the substrate binding domain. Aggregation of $0 \mathrm{~N} 4 \mathrm{R}$ tau ${ }^{\mathrm{WT}}(10 \mu \mathrm{M})$ was induced in the presence of constructs of 
the Hsc70 of Hsp72 substrate binding region (aa 391-540). The effect of chaperones on the aggregation lag time ( $\log _{2}$ fold change compared to tau alone control) is plotted for multiple chaperone concentrations tested (mean \pm SEM, 3 independent experiments). 


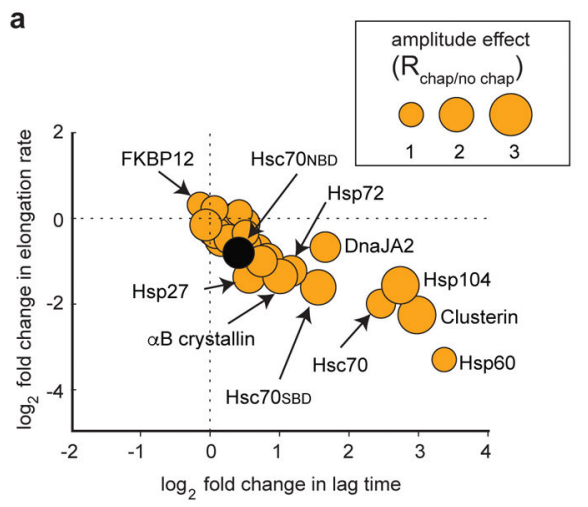

b
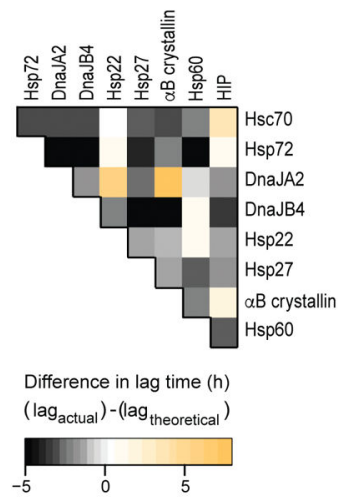

c
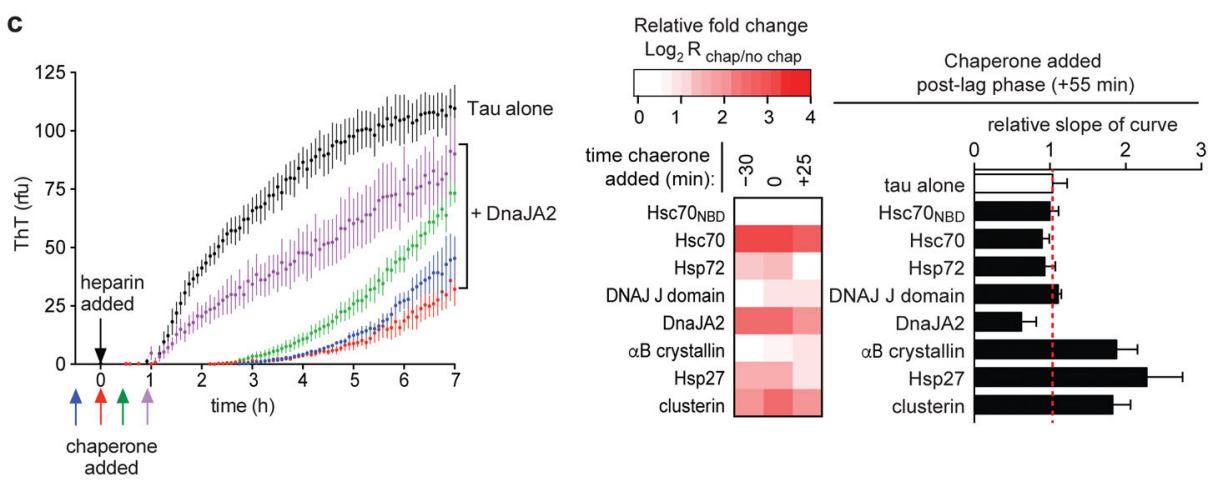

Figure 2. Unique chaperone profiles with respect to tau aggregation suggest distinct mechanisms of anti-aggregation activity

a) Summary of average effect of chaperones on tau aggregation kinetic parameters: lag time, elongation rate constant and amplitude. Each chaperone is represented by one marker plotted for its effect on each kinetic parameter averaged across all ON4R tau variants, excluding ON4R $\Delta K 280$. The $\mathrm{x}$ and $\mathrm{y}$ co-ordinates represent the lag time and elongation rate changes, respectively, as compared to tau alone controls. The size of the marker represents the change in amplitude compared to tau alone controls. b) Assaying for synergy between chaperones in delaying tau aggregation. Pairwise combinations of chaperones ( $5 \mu \mathrm{M}$ each) were assayed for their ability to increase the lag time kinetic. The difference between the observed and predicted theoretical lag time (sum of lag time effect for individual chaperones) is plotted in a heatmap with grey and orange representing observed lag times lower or higher than theoretical lag times, respectively. c) Addition of chaperones to tau aggregation assay postinduction reveals multiple mechanisms of chaperone activity. Graph depicts effect of DnaJA2 addition to tau reaction mixture (1:1 ratio) at the indicated time points (arrows) during the aggregation process (arrows and corresponding curves denoted by color, mean \pm SEM). Heatmap displays effect of chaperone addition at timepoints relative to aggregation induction on lag time parameter analyzed as described in Figure $2 \mathrm{~d}$. For time point where chaperone was added following the lag phase (+55 min, purple arrow), the representative bar graph plots the slope for the each aggregation curve relative to tau alone controls (mean \pm SEM). 


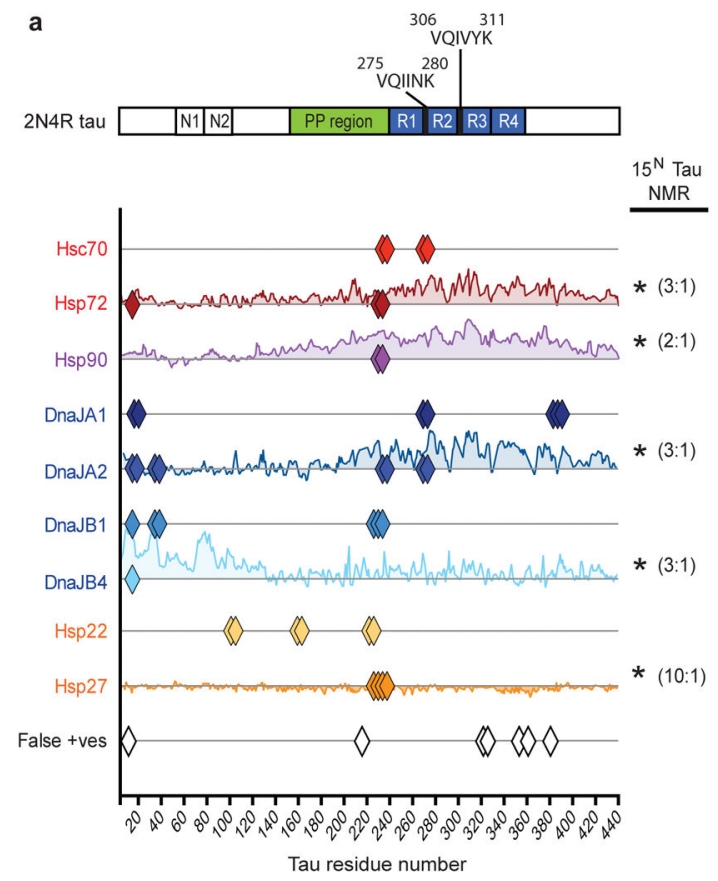

b 1) 275 - VQIIINKKLDLSNVQSKCGS - 292 2) 304 - GSVQIVYKPVDLSKVTSKC - 324

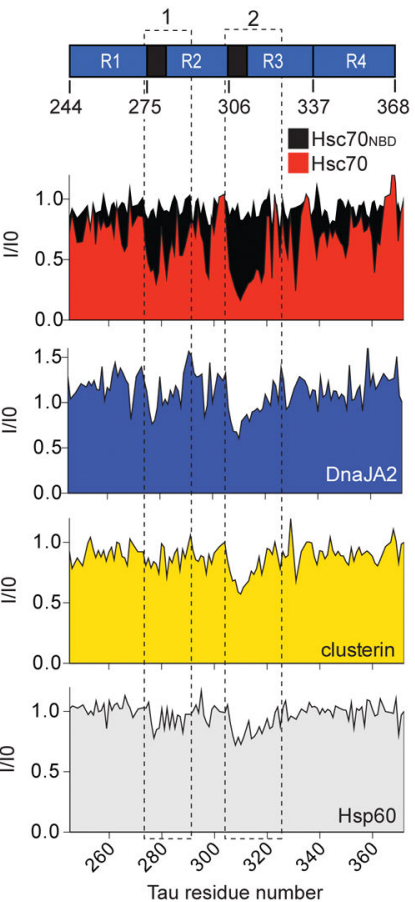

Figure 3. Anti-aggregation chaperones bind to regions spanning the tau aggregation motifs Binding sites for chaperones were identified using peptide arrays and NMR (see Methods for details). A schematic of 2N4R mapping domain and aggregation motif positions is provided for reference (see Fig. 1a for details). Location of chaperone-binding peptides from the peptide array experiments are indicated by diamonds and aligned with position in $2 \mathrm{~N} 4 \mathrm{R}$ sequence. The binding profiles on tau for selected chaperones as probed by NMR (indicated by $\left.{ }^{*}\right)$ were measured by titration of chaperones into ${ }^{1} \mathrm{H}^{15} \mathrm{~N}$ 2N4R tau. Changes in the NMR intensity ratios $\left(1-\mathrm{I} / \mathrm{I}_{0}\right)$ for individual peaks derived from HSQC spectra (I, chaperone present, $\mathrm{I}_{0}$, tau alone) are plotted, aligned with their position in the $2 \mathrm{~N} 4 \mathrm{R}$ tau sequence. Chaperones:tau molar ratios assayed are indicated in brackets.

b) Tau binding profiles of a subset of anti-aggregation chaperones identified in the screen as probed by NMR of ${ }^{15} \mathrm{~N}-\mathrm{K} 18$ tau (2:1 molar ratio chaperone:tau). Changes in NMR intensity ratios $\mathrm{I} / \mathrm{I}_{0}$ plotted as described in a). Two binding regions ( 1 and 2 , boxed by dashed lines with corresponding amino acid sequences) were identified and regions overlap with the PHF6* and PHF6 motifs (underlined). 
a

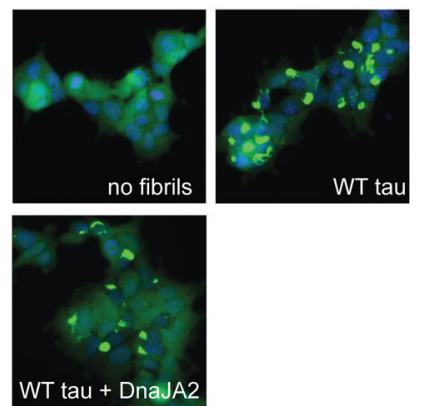

c

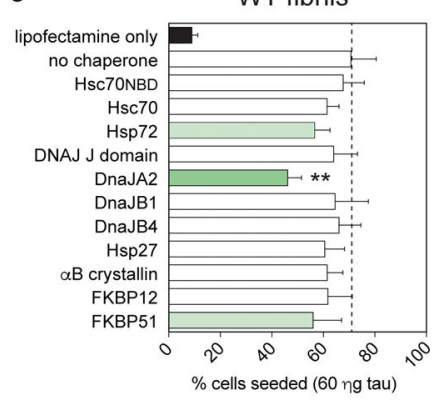

b

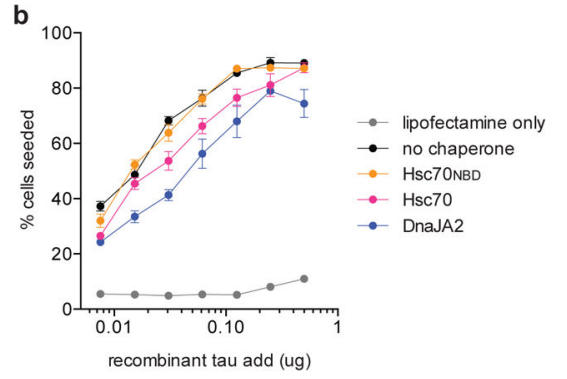

P301L fibrils

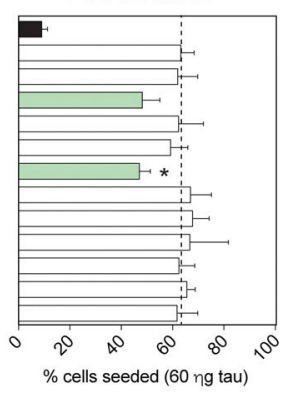

A152T fibrils

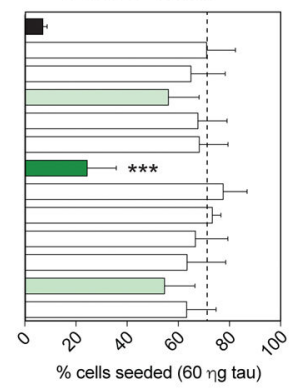

Figure 4. Tau fibrils formed in the presence of DnaJA2 have a reduced capacity to seed tau aggregation in cells

a) Representative K18-YFP fluorescence images of Clone 1 cells transfected with lipofectamine only (no fibrils), 0N4R tau ${ }^{\text {WT }}$ fibrils (60 ng), or 0N4R tau ${ }^{\text {WT }}$ fibrils (60 ng) made in the presence of DnaJA2 (WT tau+DnaJA2). b) Seeding of tau fibrils formed in the absence or presence of chaperones. The percentage of Clone 1 cells that form punctae (\% cells seeded) is correlated with the amount of aggregated tau solution ( $\mu$ g tau transfected). Unfractionated $0 \mathrm{~N} 4 \mathrm{R}$ tau ${ }^{\mathrm{WT}}$ fibrils made in the presence of the indicated chaperones were transfected and the $\%$ of cells seeded plotted (mean \pm SD of triplicates, representative of 3 independent experiments). c) Significant reduction in seeding phenotype observed for fibrils formed in the presence of DnaJA2. Unfractionated WT, P301L and A152T 0N4R tau fibrils formed in the presence or absence of the indicated chaperones were transfected into Clone 1 cells (60 $\eta \mathrm{g} \mathrm{tau}$ ) and compared by the percentage of cells seeded (mean \pm SEM, 3 independent experiments). Groups with $>20 \%$ reduction compared to the no chaperone control group are shaded in green. One-way ANOVA with a post-hoc Dunnett's test, $* \mathrm{p}=0.039, * * \mathrm{p}=0.002, * * * \mathrm{p}=0.0001$. 
a

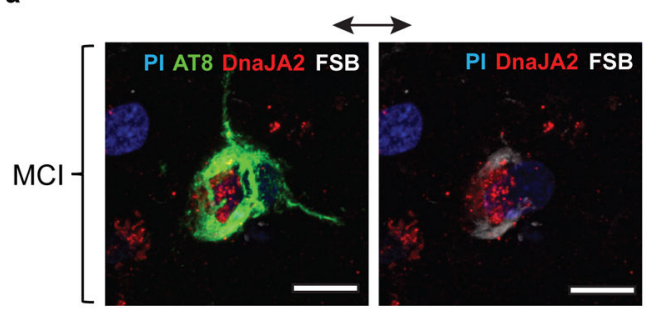

b

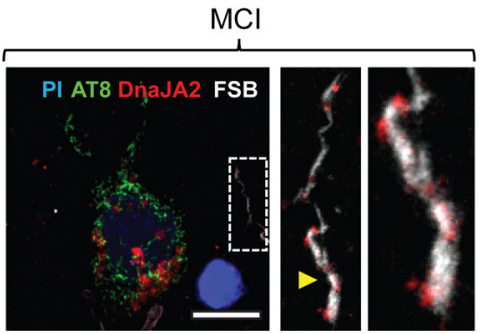

c
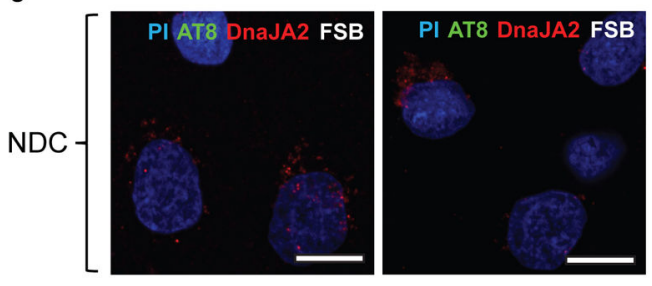

e

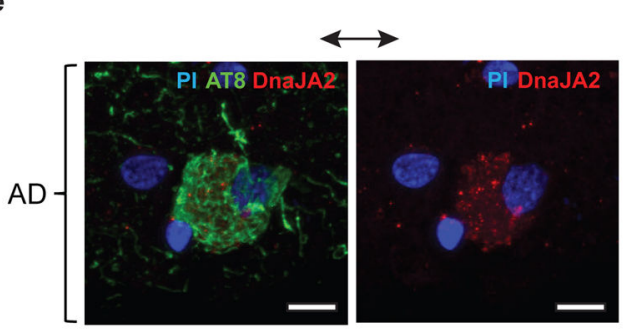

d
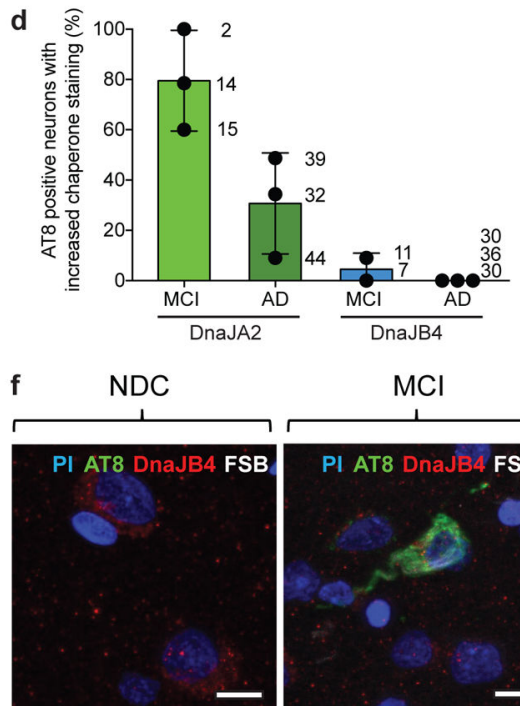

$\mathrm{MCl}$

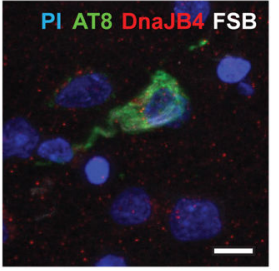

Figure 5.

DnaJA2 levels are selectively increased in neurons positive for tau pathology within MCI and late-stage AD patient samples. Fixed brain sections from MCI, late-stage AD, or nondemented control (NDC) samples were co-stained with DnaJA2 or DnaJB4(red) and phospho-tau (AT8, green) antibodies as well as the amyloid-binding small molecule, FSB (white). Nuclei were visualized with propidium iodide(PI) stain. Scale bar=10 microns for all images. a) Representative images from MCI sample of an AT8 and FSB positive neuron with increased DnaJA2 staining. AT8 staining has been excluded from one of the images for better visualization of DnaJA2 and FSB stains. b) An image of an AT8 positive neuron adjacent to an FSB positive neurite (boxed). Higher magnification images of the neurite show DnaJA2 staining along neurite with pathology. c) Representative images of low level punctate staining pattern for DnaJA2 in NDC samples. d) Increased DnaJA2 staining within AT8 positive neurons in MCI and AD samples. Individual AT8 postive neurons scored for increased DnaJA2 or DnaJB4 levels in somas relative to levels observed in non-demented control samples. The percentage of AT8 positive neurons with increased chaperone staining is plotted (bar, mean $\pm \mathrm{SD}, \mathrm{n}=$ number of cases analyzed). Multiple cases were examined for each disease type and the mean of each individual case is also plotted (black markers) with the number of neurons analyzed per case indicated to the right of each marker. e) Representative images of an AT8 positive neuron in an AD sample with increased DnaJA2 
staining. f) Images of neurons from NDC and MCI samples show no increases in DnaJB4 staining between AT8 positive and AT8 negative neurons. 